\title{
Instabilitätsformen des Schultergelenks
}

\author{
Diagnostik und Therapie
}

\author{
Sanjay Weber-Spickschen, Hamid Hosseini, Jens Agneskirchner
}

\section{Zusammenfassung}

Anatomisch bedingt sind Instabilitätsprobleme am Schultergelenk sehr häufig. Die Bandbreite reicht von der Laxizität ohne Krankheitswert bis hin zu akuten oder chronischen Luxationen mit weichteiligen und ggf. auch knöchernen Begleitverletzungen. Entsprechend der großen Vielfalt an zugrunde liegenden Entitäten unterscheiden sich die resultierenden Therapieoptionen. Um eine optimale Behandlungsstrategie abzuleiten, ist die präzise Diagnostik unerlässlich. Im folgenden Artikel geben wir einen Überblick über die relevanten anatomischen Grundlagen, fassen wesentliche Bausteine der suffizienten Diagnostik zusammen und stellen aktuelle Therapiemöglichkeiten vor. Im Fokus stehen sowohl nicht operative Maßnahmen wie die Reposition nach akuter Schulterluxation als auch spezielle Operationsmethoden wie die arthroskopische Latarjet-Operation nach rezidivierenden Luxationsereignissen und relevantem Knochenverlust.

\section{Types of Instability of the Shoulder Joint: Diagnostics and Therapy}

For anatomic reasons instability problems at the shoulder joint are very common. The spectrum ranges from laxity without clinical significance through to acute or chronic dislocations with accompanying soft-tissue and possibly also bone injuries. As a consequence of the wide range of underlying entities there are also numerous therapeutic options. In order to determine the optimal treatment strategies an exact diagnostic workup is mandatory. In the present contribution we give an overview of the relevant anatomic principles, summarise the essential components for an adequate diagnostic procedure and describe the current therapeutic options. The emphasis is placed not only on non-surgical interventions such as repositioning after acute shoulder dislocations but also on special surgical methods such as the arthroscopic Latarjet operation after dislocation recurrences and relevant bone deficits.

\section{Einleitung}

Das Schultergelenk weist im Vergleich zu den anderen Gelenken des menschlichen Körpers eine enorme Beweglichkeit in sämtlichen Freiheitsgraden auf. Um die großen Bewegungsumfänge zu gewährleisten, sind einige anatomische Besonderheiten notwendig.

Im Vergleich zum Gelenkkopf ist die Gelenkpfanne des Schultergelenks sehr klein. Nur ein Viertel des Oberarmkopfs ist von der knöchernen Pfanne umfasst.

OP-JOURNAL 2013; 29: 72-80

(c) Georg Thieme Verlag KG Stuttgart · New York DOI http://dx.doi.org/10.1055/s-0032-1328458
Um eine ausreichende Stabilität zu gewährleisten, ist das Zusammenspiel verschiedener weichteiliger Faktoren besonders wichtig. Ein wichtiger Stabilisator ist das Labrum glenoidale. Dabei handelt es sich um eine knorpelige Gelenklippe, die, ähnlich wie der Meniskus am Kniegelenk, die Gelenkkongruenz und Stabilität erhöht. Rein knöchern ist die Kavität (Form der Tiefe der Gelenkspfanne) gering. Jedoch ist der Knorpel im Randbereich der Pfanne dicker als in der Mitte. Gleichzeitig verstärkt das Labrum glenoidale zusätzlich die Gesamtkavität.

Eine gesunde Schulterpfanne hat eine birnenförmige Konfiguration. Zwischen Humeruskopf und Gelenkpfanne wirkt ein minimaler Unterdruck. Dieses leichte
Vakuum kann nur dann bestehen, wenn das umgebende Labrum intakt ist. Die wichtigsten Strukturen für die statische Stabilisierung sind jedoch die glenohumeralen Bänder. Es werden 1 superiores, 1 mittleres, und 2 inferiore glenohumerale Bänder (IGHL) unterschieden. Besonders das vordere IGHL ist biomechanisch für die Luxationsrichtung nach vorne unten entscheidend, das mittlere glenohumerale Ligament ist ebenfalls ein wichtiger Stabilisierungsfaktor.

Aufgrund von akuten oder chronischen Verletzungen, aber auch anlagebedingt kann das Zusammenspiel der verschiedenen stabilisierenden Faktoren gestört sein. Dies kann zu Beschwerden führen. Im Folgenden werden wir auf verschiedene Formen eingehen und Diagnostikund Therapieoptionen vorstellen.

\section{Was bedeutet Schulterinstabilität?}

Die Schulterinstabilität wird häufig als Sammelbegriff dafür verwendet, dass der Oberarmkopf nicht zentral in der Schultergelenkspfanne positioniert ist. Von der Instabilität abzugrenzen ist die sog. Laxizität, welche als Normvariante nicht pathologisch einzustufen ist. Hierbei handelt es sich um eine im Vergleich zur Normalbevölkerung vermehrte Beweglichkeit und Translation des Oberarmkopfs im Verhältnis zur Pfanne. Häufig findet sich diese Laxizität auch an anderen Gelenken des Patienten, d.h. mehrere Gelenke des Körpers sind u.U. überstreckbar. Solange keine Symptome bestehen, liegt kein Krankheitswert vor, es liegt also lediglich eine Laxizität vor.

Streng von der Laxizität abzugrenzen ist die Instabilität, die auf dem Boden einer vermehrten Translationsfähigkeit zwischen Oberarmkopf und Glenoid Symptome verursacht. Diese Symptome können eine Subluxation oder Luxation sein. Bei der Subluxation kommt es „beinahe“ zum Auskugeln der Schulter, d.h. der Oberarmkopf ist bis an den Pfannenrand 


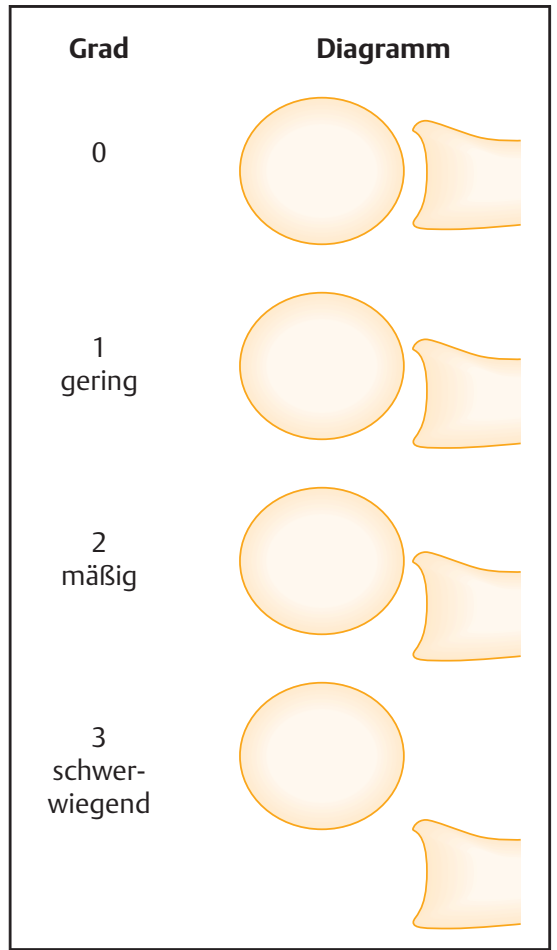

Abb. 1 Graduierung der glenohumeralen Translation durch Quantifizierung der Beweglichkeit des Oberarmkopfs in der Gelenkpfanne (nach Hawkins).

verrutscht. Bei der Luxation kommt es zum kompletten Kontaktverlust zwischen dem Oberarmkopf und der Gelenkspfanne (siehe Abb. 1).

Ursächlich für die Subluxation oder Luxation kann ein akutes Ereignis wie ein Unfall oder ein Krampfanfall sein. Gleichzeitig kann eine Luxation auch durch ein Bagatelltrauma entstehen, wenn stabilisierende Faktoren angeboren nicht ausreichend vorhanden sind oder nach vorangegangenem Unfall dauerhaft geschwächt sind (siehe Abb. 2 und 3).

Bei der Schulterinstabilität ist also das Verhältnis von Belastung zur Belastbarkeit verschoben. Die dezentrierenden Kräfte sind für die stabilisierenden Faktoren zu groß.

\section{Die akute Luxation}

Durch einen Unfall, z. B. einen Sturz oder Sportunfall, wird der Oberarmkopf durch eine große Krafteinwirkung aus der Gelenkpfanne herausgedrückt. Fast immer wird der Oberarmkopf nach vorne gepresst (anteriore Luxation), die Luxation nach hinten ist wesentlich seltener.

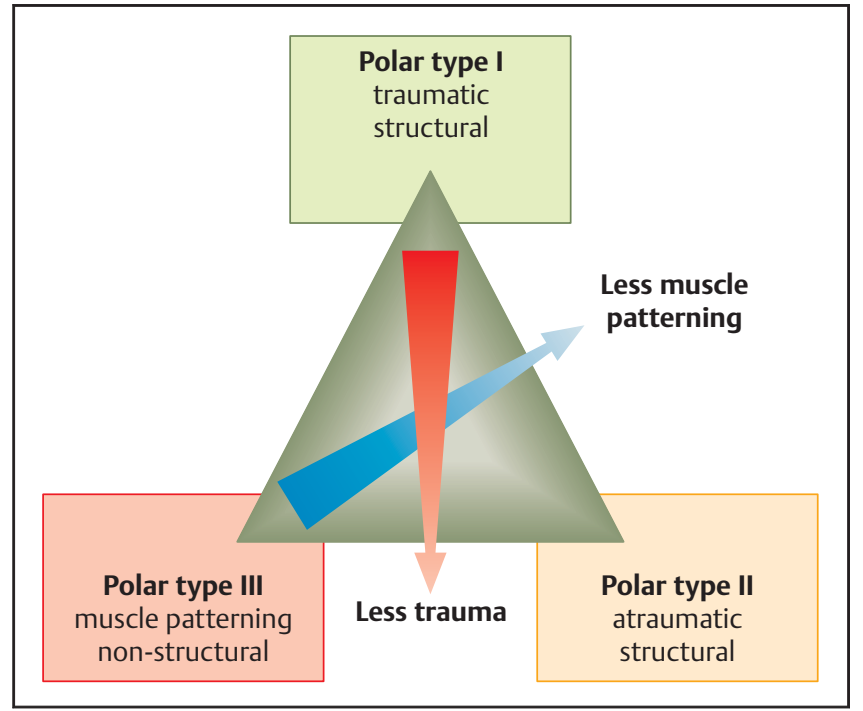

Abb. 2 Einteilung der Schulterinstabilität nach Bayley in 3 Gruppen (Polar IIII). Gezeigt ist der fließende Übergang zwischen traumatisch-strukturellen (gut zugänglich für operative Therapien), atraumatisch-strukturellen (ggf. operativ therapierbar) und nicht strukturellen (durch pathologische Muskelanspannung verursacht $=$ in der Regel nicht operativ therapierbar) Instabilitätsformen.

Die Gelenkpfanne ist nun leer, der Oberarmkopf ist oft vor der Pfanne unten verklemmt. Beim Herausrutschen des Kopfes kann es zu einem Abbruch der vorderen unteren Gelenkpfanne kommen (knöcherne Bankart-Läsion). Es kann zum Zerreißen der Kapsel und weiterer weichteiliger Strukturen kommen. Gleichzeitig ist auch möglich, dass der Humeruskopf eine knöcherne Impression im Sinne eine Delle erhält (HillSachs-Delle). Gerade beim älteren Patienten ist es möglich, dass durch die Luxation die Rotatorenmanschette reißt.

Die wichtigsten weichteiligen Stabilisatoren nach vorne und unten, das mediale glenohumerale Ligament (MGHL) und das anteriore inferiore glenohumerale Ligament (alGHL), sind bei der Luxation nach vorne regelhaft verletzt.

Kommt es zum Reißen des Labrums, ist ein weiterer wichtiger Stabilisator geschädigt und das Risiko von Re-Luxationen sehr groß.

Häufig nimmt der Patient nach stattgehabter Luxation eine typische Schonhaltung ein. Das Schultergelenk ist nur noch minimal beweglich und meist sehr schmerzhaft. In manchen Fällen kann der Patient durch eine bestimmte Bewegung die Schulter selbst reponieren. Dies ist v.a. bei Patienten mit anlagebedingt geschwächten Stabilisatoren oder nach wiederholten Luxationen möglich. Bei jungen und kräftigen Patienten mit einer traumatischen Erstluxation muss die Reposition in der Regel durch den Arzt durchgeführt werden. Hierfür gibt es verschiedene Techniken, auf die wir im Folgenden noch eingehen werden. Teil-

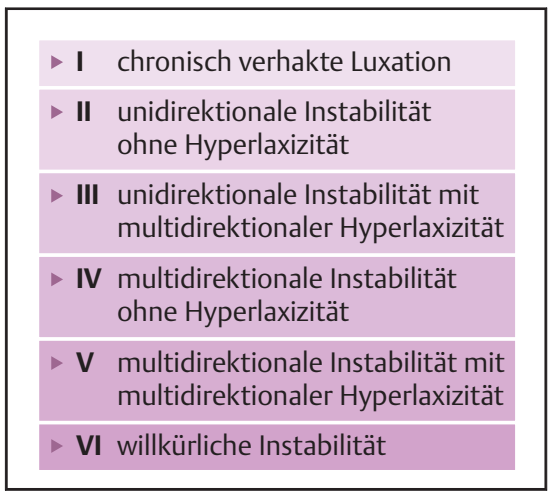

Abb. 3 Einteilung der Schulterinstabilitäten (nach Gerber, Nyffeler 2002).

weise ist die Reposition deutlich erschwert und der Patient hat starke Schmerzen. In diesen Fällen muss auf eine Kurznarkose zurückgegriffen werden, um die luxierte Schulter so schonend wie möglich zu reponieren.

Zur Bestätigung der Diagnose Schulterluxation und zum Ausschluss von Begleitverletzungen sollte in der Klinik eine Bildgebung erfolgen. Insbesondere Brüche des Oberarmkopfs und Oberarms oder Nerven- und Gefäßläsionen müssen vor einem Repositionsversuch ausgeschlossen werden (siehe Abb. 4-6).

Deutlich seltener ist die hintere Luxation, welche ebenfalls vor einem Repositionsversuch im Röntgenbild bestätigt werden sollte.

\section{Epidemiologie}

Anatomisch bedingt luxiert das Schultergelenk im Vergleich zu den anderen Gelenken des menschlichen Körpers am 
häufigsten. Männer sind insgesamt 3mal häufiger von Schulterinstabilitäten betroffen (Hovelius 1996).

Die Luxation nach vorne zeigt eine Häufigkeit von über 95\%, die Luxation nach hinten zeigt eine Häufigkeit von unter $5 \%$.

Präklinisch wichtig ist die Abgrenzung von der Schulterluxation zur Humerusfraktur. Gerade beim älteren Patienten sind subkapitale Humerusfrakturen sehr häufig. Gleichzeitig haben ältere Patienten bei einer Schulterluxation oft eine begleitende Rotatorenmanschettenruptur, nach der ebenfalls gefahndet werden muss.

Beim jüngeren Patienten sind begleitende Rotatorenmanschettenrupturen oder subkapitale Humerusfrakturen eher selten. Allerdings stellt die Gefahr der Reluxation ein großes Problem dar. So ist die Wahrscheinlichkeit einer Reluxation beim Jugendlichen und jungen Erwachsen sehr hoch.

Bei hohem Risiko der Reluxation von 94\% für unter 20 -jährige Patienten nach primär traumatischer Erstluxation und gesunder Gegenschulter ist eine operative Therapie schon nach der 1 . Luxation empfehlenswert.

Die Reluxationsrate für die 21- bis 30 Jährigen liegt bei knapp $80 \%$, die Rate für die 31 - bis 40 -jährigen Patienten bei $50 \%$. Je nach sportlicher Aktivität kann die Reluxationsrate deutlich erhöht werden. Kommt es zu Reluxationen, ist die Gefahr der weiteren Rezidive sehr hoch und eine Operation ebenfalls zu empfehlen.

Die dorsale Schulterinstabilität ist deutlich seltener als die ventrale. Sie tritt ebenfalls in 2 Erscheinungsformen auf, häufiger ist die atraumatische/habituelle Instabilität, sehr selten ist die traumatische, dorsal verhakte Luxation.

\section{Nervenschäden}

In der Literatur werden Häufigkeiten für Verletzungen des N. axillaris von bis zu $14 \%$ angegeben (Walch 1993). Diese finden sich vor allem bei älteren Patienten. $\mathrm{Zu}$ unterscheiden sind temporäre Nervenschäden des $\mathrm{N}$. axillaris mit vorübergehenden Schädigungen, von dauerhaften Schädigungen oder gar Plexusschäden.
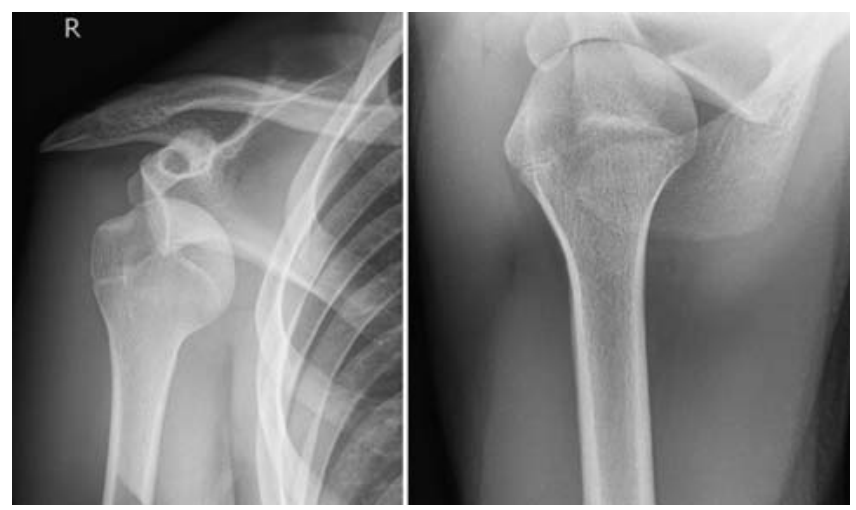

Abb. 4 Röntgen a.-p. und axial bei anteroinferiorer Schulterluxation.

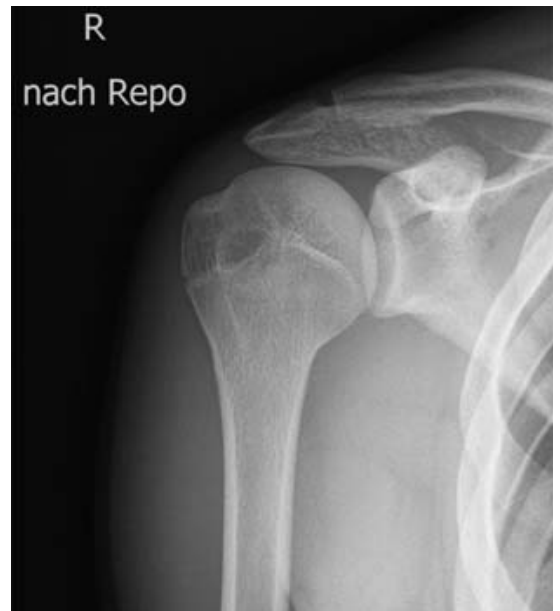

Abb. 5 Obligat nach der Reposition: erneute Röntgenkontrolle (Patient identisch zu Abb. 4).

\section{Reposition}

Nach erfolgter Akutdiagnostik sollte die luxierte Schulter zügig reponiert werden. Hierfür stehen verschiedene Techniken zur Verfügung. Von wiederholt brüsken Repositionsmanövern des unerfahrenen Therapeuten sollte dringend abgeraten werden, da bei deutlicher Schmerzauslösung ein Gegenspannen des Patienten verursacht, die Reposition erschwert wird und das Auftreten von Begleitverletzungen durch die Repositionsversuche deutlich erhöht wird.

Je nach Patient gibt es unterschiedliche Repositionstechniken mit Vor- und Nachteilen.

\section{Selbstreposition}

Bei Patienten, die gut mitarbeiten können, kann der Versuch der Selbstreposition unternommen werden. Eine ruhige Atmosphäre und die Geduld von Patient und Arzt sind unabdingbare Notwendigkeit dieser Technik.

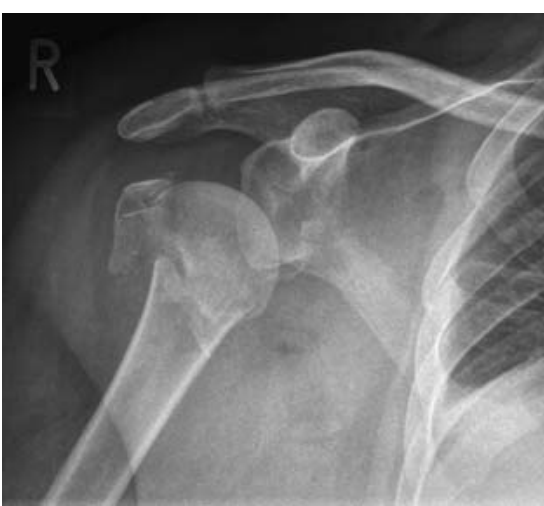

Abb. 6 Luxationsfraktur des Oberarmkopfs: Kombination aus Oberarmkopffraktur und anteroinferiorer Glenoidfraktur.

Der Patient liegt mit angezogenen Beinen auf der Untersuchungsliege. Beide Hände werden vor den Kniegelenken mit einer Schlinge fixiert. Nun wird der Patient aufgefordert, sich zu entspannen und sich ganz langsam zurückzulehnen. Die Hüfte soll dann ganz langsam gestreckt werden und der Patient sich millimeterweise nach hinten lehnen. Der gesamte Vorgang dauert einige Minuten und soll so schmerzarm wie möglich durchgeführt werden. Nach einigen Minuten kommt es zur Selbstreposition. Gibt der Patienten vor der Reposition starke Schmerzen an, sollte der Versuch abgebrochen werden. Durch den Schmerz kommt es zum Anspannen der Muskulatur, was die Reposition verhindert.

Gerade bei sehr ängstlichen, schmerzgeplagten und eher wenig kooperativen Patienten sollte die Reposition in Analgosedierung erfolgen.

\section{Fremdreposition}

Unter Kreislaufüberwachung und Analgosedierung kann die Methode von Hippokrates verwendet werden. Hierbei wird die Ferse des Arztes in der Achsel- 

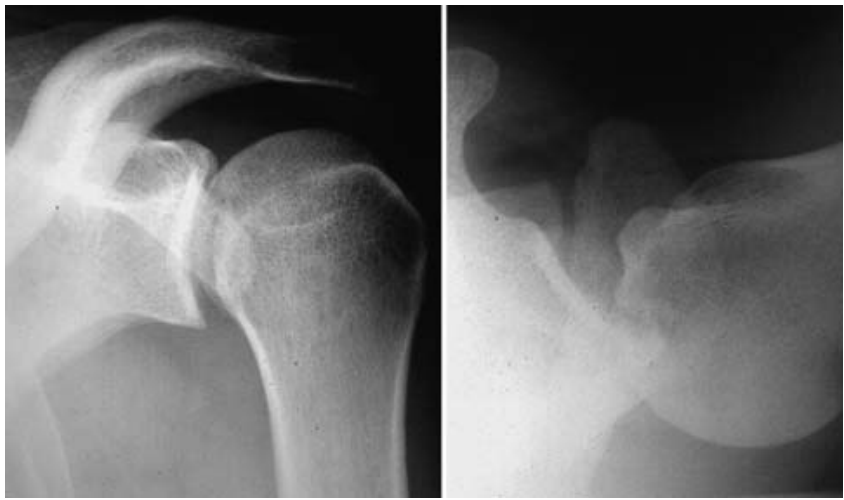

Abb. 7 Verhakte dorsale Schulterluxation im a.-p. und axialen Röntgenbild.

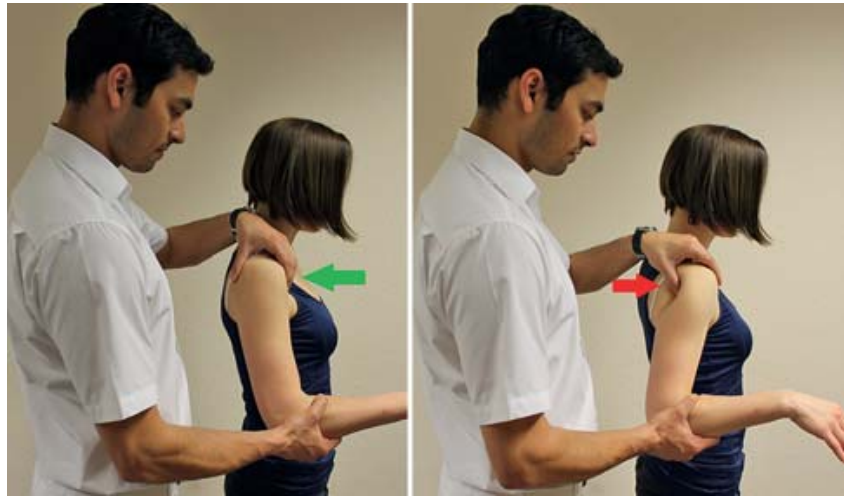

Abb. 8 Klinische Überprüfung der anteroposterioren Translation im Glenohumeralgelenk.

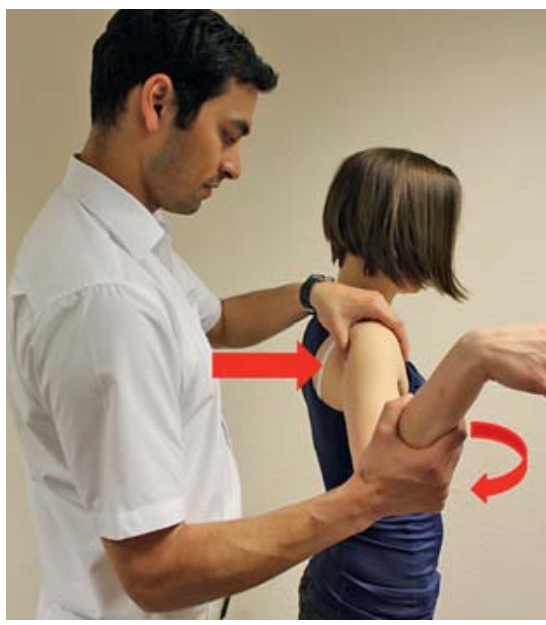

Abb.9 Klinischer Test zur Überprüfung der vorderen Instabilität: Apprehension-Test.

höhle der betroffenen Schulter des Patienten positioniert und langsam ein Zug am Arm aufgebaut. Bei dieser Methode sind Plexusläsionen durch $\mathrm{zu}$ brüskes Reponieren beschrieben worden. Ohne Analgosedierung kann diese Repositionsmethode Angst beim Patienten erzeugen, was einen erfolgreichen Repositionsversuch ebenfalls verhindern kann.

Nach erfolgreicher Reposition sollten erneut Durchblutung, Motorik und Sensibilität überprüft werden. Weiterhin sollte eine bildgebende Diagnostik (insbesondere Röntgenuntersuchung, siehe Abb. 5) erfolgen.

In den ersten Tagen nach Reposition sollte eine Ruhigstellung in leichter Außenrotation durchgeführt werden. Eine länger dauernde Immobilisierung (mehr als 1 Woche) des Schultergelenks ist in der Regel nicht erforderlich oder sinnvoll.

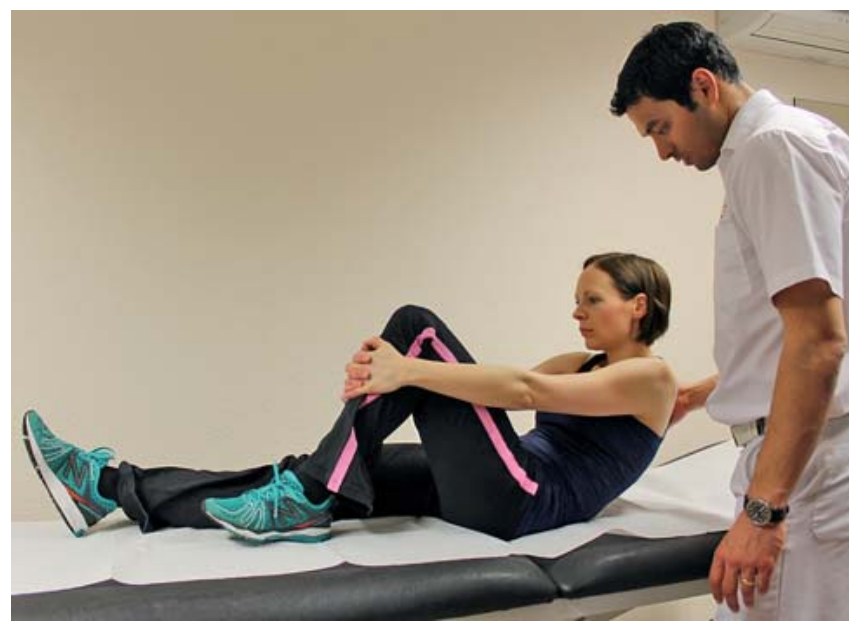

Abb. 10 Methode zur Reposition der Schulterluxation (Eigenrepositionsmanöver).

\section{Traumatisch, dorsal verhakte Luxation}

Diese kann durch einen Sturz auf den nach vorne ausgestreckten Arm verursacht werden [1]. Eine besondere Bedeutung liegt im Erkennen dieser Luxationsform, da sie zum einen selten ist und zum anderen sehr häufig übersehen wird.

Das klinische Erscheinungsbild ist teilweise nicht so eindeutig, hochgradig verdächtig ist eine fehlende Außenrotation des Patienten. Radiologisch erscheint in der a.-p. Röntgenaufnahme der Humeruskopf in einer birnenförmigen Konfiguration. In der axialen Aufnahme ist die nach dorsal luxierte Schulter in der Regel leicht zu erkennen (Abb. 7).

Die dorsal luxierte Schulter ist in der Regel schwieriger zu reponieren und meist nicht durch Verfahren ohne Analgosedierung möglich. Nach Reposition erfolgen auch bei der dorsalen Luxation die Kontrolle von Durchblutung, Motorik und Sensibilität sowie die radiologische Kontrolle. Häufig zeigt sich eine Reversed-Hill-Sachs-Delle am Humerus-

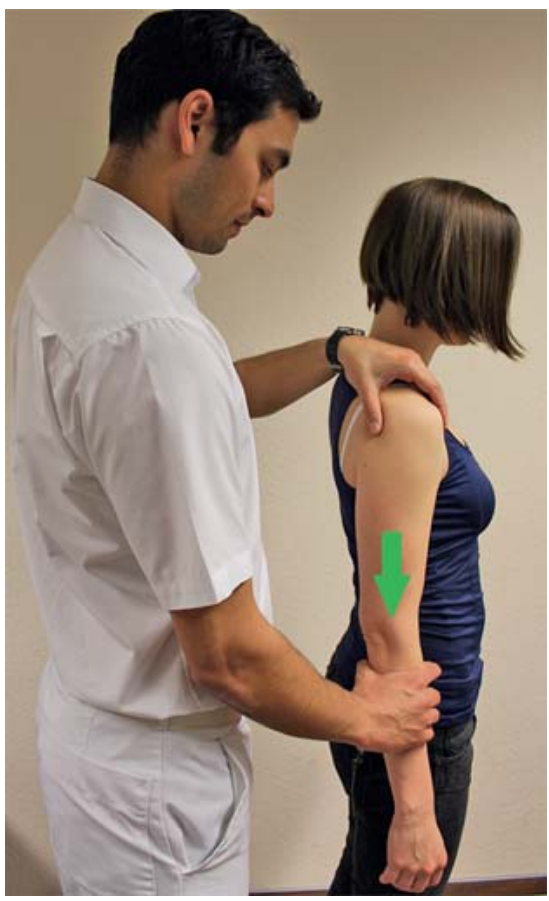

Abb. 11 Klinischer Test zur Überprüfung des Rotatorenintervalls: Sulcus-Zeichen. 
kopf. Die Größe der Impression bedingt die OP-Indikation. Genauere Aussagen lassen sich in der Regel mithilfe einer Computertomografie treffen. Bei kleinen Impressionen ist eine konservative Therapie mit temporärer Ruhigstellung möglich. Bei mittleren Impressionen zwischen 20 und $40 \%$ der Gelenkfläche sollte im akuten Fall die Impression durch Aufstößelung oder Unterfütterung mit Spongiosa durchgeführt werden.

\section{Atraumatische dorsale Instabilität}

Die atraumatische/habituelle dorsale Instabilität ist deutlich häufiger als die akut traumatische dorsale Luxation. Besonders häufig ist die dorsale Instabilität mit einer weiteren Instabilitätsrichtung kombiniert. In diesem Falle spricht man von einer multidirektionalen Instabilität [6]. Die multidirektionale Instabilität darf nicht mit einer Hyperlaxität verwechselt werden, welche symptomfrei keinen Krankheitswert hat (siehe oben). Patienten mit multidirektionaler Instabilität und Hyperlaxität sollten zunächst intensiv konservativ behandelt werden. Kräftigungs- und Stabilisierungsübungen müssen hierfür regelmäßig mindestens 2-3-mal die Woche über einen mindestens 1/2-jährlichen Zeitraum durchgeführt werden. Führt die konservative Therapie zu keinem Erfolg, können operative Maßnahmen eingesetzt werden. Wie erwähnt spricht man dann von einer Instabilität, wenn der Patient nicht mehr in der Lage ist, seine Schulter in bestimmten Stellungen $\mathrm{zu}$ kontrollieren und deshalb Beschwerden hat.

\section{Klinische Diagnostik}

Bei der chronischen/habituellen vorderen Instabilität berichten die Patienten meist über große Angst vor Luxationen im Alltag. Oft reichen bestimmte Alltagsbewegungen aus, um eine Sub-/Luxation hervorzurufen.

Klinisch sollten immer beide Schultern vergleichend untersucht werden.

\section{Sulcus-Zeichen}

Der Untersucher zieht den lockeren hängenden Arm des Patienten nach bodenwärts. Typischerweise lässt sich der Oberarmkopf aus der Gelenkpfanne nach unten bewegen, was sich an einer vermehrten Bewegung und einer tastbaren Einziehung an der seitlichen Schulter zeigt (siehe Abb. 11).

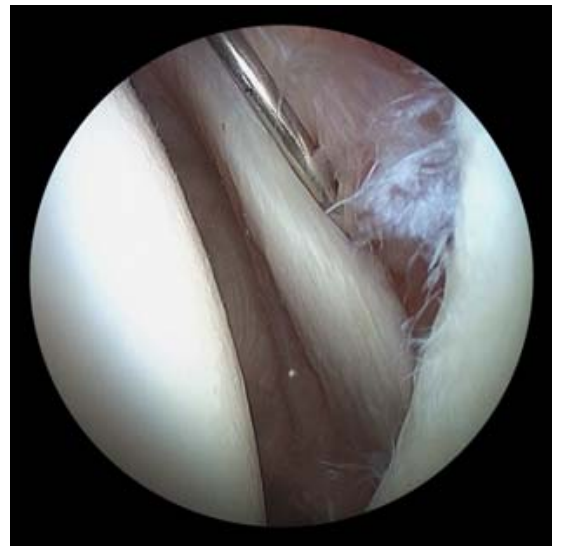

Abb.12 Arthroskopisches Bild bei BankartLäsion (linke Schulter, Blick von posterior): Tasthaken zwischen abgerissenem anterioren Labrum und Pfannenrand.

\section{Vermehrte anteriore-posteriore} Beweglichkeit

Der Untersucher steht hinter dem Patienten und hält den Oberarmkopf zwischen Daumen und Mittelfinger. Nun drückt er mit dem Daumen den Oberarmkopf nach vorne aus der Gelenkpfanne und anschließend mit dem Mittelfinger nach hinten aus der Gelenkpfanne (Abb. 8). Ein leichtes Gelenkspiel von bis zu $8 \mathrm{~mm}$ ist physiologisch. Wichtig ist es, darauf zu achten, ob im Seitenvergleich oder in einer Richtung eine deutlich vergrößerte Verschieblichkeit vorliegt.

\section{Apprehension-Test}

Hierbei wird die eine Hand des Untersuchers ebenfalls im Codmann-Handgriff auf die zu untersuchende Schulter gelegt. Mit der 2. Untersucherhand wird der Arm des Patienten in die maximal mögliche Außenrotationsposition bewegt. Nun wird in den Abduktionspositionen $0^{\circ}, 30^{\circ}, 60^{\circ}, 90^{\circ}$ und $120^{\circ}$ nach „Angst“ (Apprehension) oder Schmerzen bei der jeweilig vorliegenden Armposition gefahndet, ggf. kann durch leichten Druck von dorsal auf den Oberarmkopf der Stress noch verstärkt werden(Abb. 9).

\section{Bildgebende Diagnostik}

Sowohl bei der akuten Luxation als auch bei der Diagnostik chronischer Instabilitäten bei Patienten mit wiederholten Verrenkungen sollten obligat Röntgenaufnahmen in mindestens 3 Ebenen durchgeführt werden: true a.-p., axial (ggf. Bernageau), y-view. Neben proximalen Humerusfrakturen sind Luxationsrichtung, das Vorliegen einer Hill-
Sachs-Delle und die knöcherne BankartLäsion von Interesse (siehe Abb. 4-7).

In der Ultraschalluntersuchung sollte nach Läsionen der Rotatorenmanschette gefahndet werden. Diese treten mit zunehmender Häufigkeit beim älteren Patienten auf, können aber auch akut traumatisch beim jüngeren Patienten vorhanden sein. Insbesondere die akute Läsion der Subscapularissehne stellt eine sehr wichtige OP-Indikation dar. Die Refixation der Subscapularissehne sollte so zeitnah wie möglich erfolgen. Sie hat eine zentrale Bedeutung für die dynamische Stabilität in der Schulter.

Die Kernspintomografie kann die Lokalisation und das Ausmaß des Weichteilschadens erfassen und ist insbesondere bei der akuten Luxation indiziert. Läsionen der Gelenklippe („Bankart-Läsion“), der Gelenkkapsel und des Bandapparats sind bei entsprechender Aufnahmetechnik zu sehen.

Die Diagnostik knöcherner Defekte an der Pfanne und am Oberarmkopf, die bei chronischen Instabilitäten nicht selten sind, erfolgt am besten in der Computertomografie. Mittels moderner 2-D- oder 3-D-Rekonstruktion können relevante Defekte, welche im Röntgen oder MRT ggf. unterschätzt werden, sehr anschaulich und exakt quantitativ erfasst werden.

\section{Operative Therapie der Schulterinstabilität}

\section{Operationsziel}

Die klassische Bankart-Läsion ist das typische Verletzungsmuster bei der vorderen Schultergelenksluxation. Hierbei kommt es zu einem Abriss des vorderen Kapsel-Labrum-Komplexes vom Pfannenrand (siehe Abb. 12). Ziel der Operation ist, den abgerissenen Kapsel-Labrum-Komplex wieder am Glenoidrand zu refixieren. Die Ergebnisse moderner arthroskopischer Verfahren der Bankart-OP sind mit denen der offenen Technik vergleichbar.

Kontraindikationen für die BankartOperation sind ein relevanter Knochenverlust an Gelenkpfanne oder Kopf, eine schlechte Kapselqualität, sog. „HAGL-Läsionen“ (Kapselverletzungen am Oberarmkopf). 


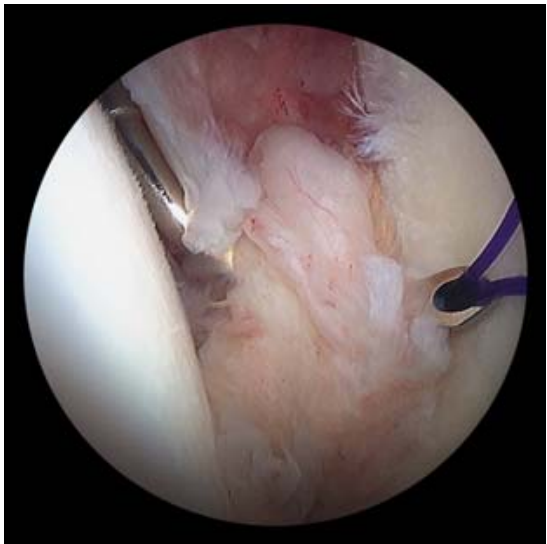

Abb. 13 Kapselshift durch Durchstechen des mobilisierten Kapsel-Labrum-Gewebes mittels eines kanülierten Nadelsystems.

\section{OP-Technik}

\section{Anästhesie/Lagerung}

Die Patienten werden einer Allgemeinanästhesie mit Intubationsnarkose zugeführt. Sowohl die Seitenlagerung als auch die Beach-Chair-Position sind mögliche Lagerungsformen für den arthroskopischen Eingriff. Zunächst wird eine Narkoseuntersuchung durchgeführt. Die vermehrte Translation des Oberarmkopfs sollte in alle Richtungen geprüft werden. Anschließend sollte darauf geachtet werden, ob ein positives SulcusZeichen als Zeichen einer Insuffizienz des Rotatorenintervalls vorliegt.

In unserer Klinik verwenden wir die Beach-Chair-Position mit Lagerung des Arms in einem Armhalter (z.B. Spider, Fa. Smith \& Nephew). Der Arm wird in Neutralrotation ohne wesentlichen Längszug gelagert.

Instrumente und Implantate für die arthroskopische Bankart-Operation

- Spülpumpe, Shaver, Radiofrequenzinstrumente

- scharfes Elevatorium und Raspatorium

- kanülierte, gebogene Nadeln mit verschiedenen Richtungen

- Fadenankersystem

- ggf. Knotenschieber

- optional Arbeitskanülen (Durchmesser $8 \mathrm{~mm}$ )

\section{Operationstechnik}

Nach gründlicher steriler Desinfektion und Abdeckung werden die knöchernen Landmarken eingezeichnet. Es werden 3 Standardportale angelegt. Ein dorsales Standardportal für das Arthroskop, ein anterosuperiores Arbeitsportal durch

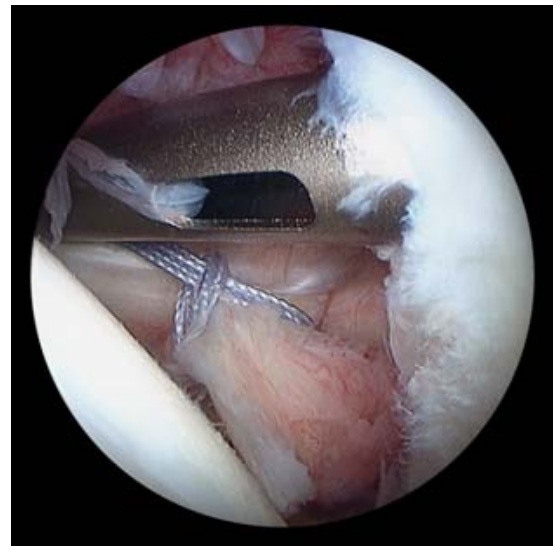

Abb. 14 Anschlingen des Gewebes mit Fiberwire-Faden (Cinch-Stitch-Technik), dann Vorbohren für einen knotenlosen Anker.

das Rotatorenintervall und ein anteroinferiores Portal zur Ankerpositionierung direkt oberhalb der Subscapulrissehne. Der Eintritt der vorderen Zugänge wird unter Sichtkontrolle mit einer Braunüle kontrolliert. Zunächst wird ein glenohumeraler diagnostischer Rundgang des ventralen Gelenkabschnitts durchgeführt. Neben Kapsel-Labrum-Verletzungen sollte dabei auf das Rotatorenintervall geachtet werden. Falls nötig kann eine dynamische Untersuchung nach Portalwechsel erfolgen.

\section{Kapsel-Labrum-Rekonstruktion}

Die klassische Bankart-Läsion beschreibt den Abriss des vorderen Kapsel-LabrumKomplexes vom vorderen Pfannenrand von der 2-Uhr- bis zur 5-Uhr-Position. Ziel ist nun die Refixation des abgerissenen Kapsel-Labrum-Komplexes mittels Fadenankern am Pfannenrand.

Da das sog. inferiore glenohumerale Ligament (IGHL) als wichtigster statischer Stabilisator weit inferior an der Kapsel inseriert, ist der erste inferiore Anker der entscheidende, da hierüber der Kapselshift und die Rekonstruktion des Bandapparats erreicht wird.

Beim Vorliegen einer Bankart-Läsion wird der Kapsel-Labrum-Komplex mit einem Elevatorium bis weit nach medial und inferior vom Glenoidrand mobilisiert. Dieser Schritt muss sehr gründlich durchgeführt werden, dass alle Adhäsionen zwischen Kapsel-Labrum-Komplex und Knochen gelöst werden. Bei Vorliegen einer sog. ALPSA-Läsion (anterior labrum periostal sleeve avulsion) kann optional ein zusätzliches Portal lateral der langen Bizepssehne angelegt werden (suprabizipitales Portal). Bei diesen Lä-

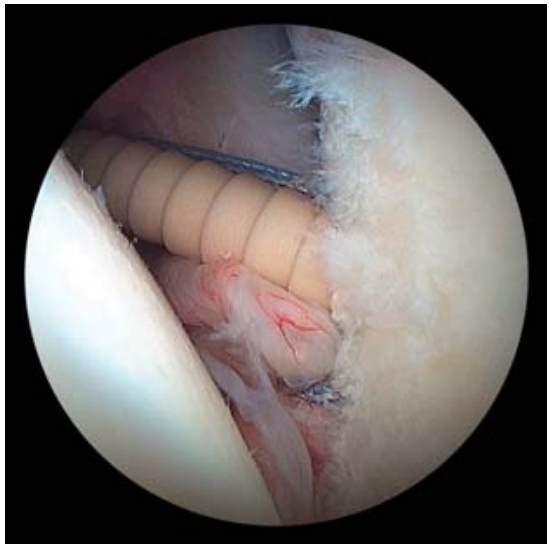

Abb. 15 Einschlagen eines knotenlosen Ankers (pushlock PEEK 3,5 mm).

sionen ist das Kapsel-Labrum-Gewebe am Glenoidhals u.U. weit medial vernarbt und kann mit dem Arthroskop von dorsal schlecht eingesehen werden. Mit dem Arthroskop im suprabizipitalen Portal kann die ALPSA-Pathologie viel besser visualisiert und die Mobilisation besser kontrolliert werden. Anschließend wird die Glenoidkante mit einem Bankart-Raspatorium oder einem motorisierten Shaver angefrischt, um eine fibroblastische Einheilung zu gewährleisten. Das mobilisierte Kaspel-Labrum-Gewebe wird nun mit einem kanülierten Nahthaken geeigneter Krümmung durchstochen und ein Shift nach kranial und medial durchgeführt (Abb. 13). Dabei muss das IGHL perforiert werden, um einen ausreichenden Kapselshift zu ermöglichen. Eine alleinige Labrumrekonstruktion ist nicht ausreichend, da es über den verbliebenen Kapselpouch erneut zu Luxationen kommen kann. bei Ein monofiler Faden (PDS USP 2-0) wird durch die Nadel geschoben, der Nahthaken entfernt und die nun intraartikulär liegenden Fäden mit einem Fadenholer erneut durch das anteroinferiore Portal ausgeleitet. Mithilfe dieses PDS Shuttle Relay wird ein Fiberwire-Faden durch den mobilisierten Kapsel-Labrum-Komplex transportiert. Mit dem Fadenholder werden beide Enden des Fiberwire-Fadens durch das anteroinferiore Portal ausgeleitet. Dabei werden die Enden durch die Schlaufe des Fadens herausgezogen. Für die Ankerpositionierung wird nun über eine eine Führungsbuchse ein Bohrloch in einem $45^{\circ}$-Winkel zur Glenoidlängs- und -querachse über das anteroinferiore Portal weit inferior angelegt (Abb. 14). Der Vorteil des vorgelegten Faden (suture first-Technik) besteht nun darin, dass durch dosiertes Anspannen der Fäden kontrolliert wer- 


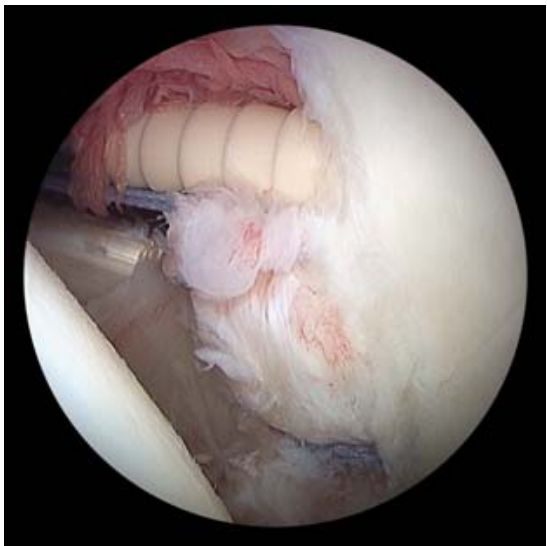

Abb. 16 Fortführen der Kapselrekonstruktion durch schrittweises Arbeiten von inferior nach superior mit Refixation durch weitere Anker.

den kann, wo genau die Ankerplatzierung erfolgen muss, um einen ausreichenden Kapselshift zu erreichen. Nach Anlage der Bohrung wird die Führungshülse entfernt. Beide Enden des Fadens werden durch die Öse des Ankers außerhalb des Gelenks eingefädelt und diese unter dosiertem Anspannen des Fadenpaars in das zuvor angelegte Bohrloch eingeschlagen. Die Fäden werden anschließend gekürzt (Abb. 15). Durch Anlage des ersten Ankers kommt es durch den Kapselshift zu einer signifikanten Reduktion des Kapselvolumens. In identischer Technik werden nun weitere Anker nach Durchstechen des Kapsel-Labrum-Komplexes angelegt (Abb. 16). In der Regel werden 2-3 Anker in der 5Uhr-, 3-Uhr- und ggf. 1-Uhr-Position angelegt.

\section{Rehabilitation}

Die Patienten sollten zunächst in einer Schulterorthese (z.B. Medi Arm Fix) für die ersten 6 postoperativen Wochen immobilisiert werden. Innerhalb der ersten 6 Wochen kann die Abduktion und Flexion bis $60^{\circ}$ freigegeben werden. Die Außenrotation ist bei $0^{\circ}$ limitiert. Nach 6 Wochen kann das Bewegungsausmaß sukzessiv gesteigert werden, bis eine freie glenohumerale Beweglichkeit erreicht wird. Zuletzt sollten auch die Rotatorenmanschette, der M. deltoideus und die Scapulastabilisatoren auftrainiert werden. Überkopf- oder Kontaktsport sollte für die Dauer von 6 Monaten nicht durchgeführt werden.

\section{Hintere Instabilität}

Die hintere Schulterinstabilität ist mit 2$5 \%$ aller Instabilitätsformen eine seltene Entität. Im Gegensatz zur meist trauma-
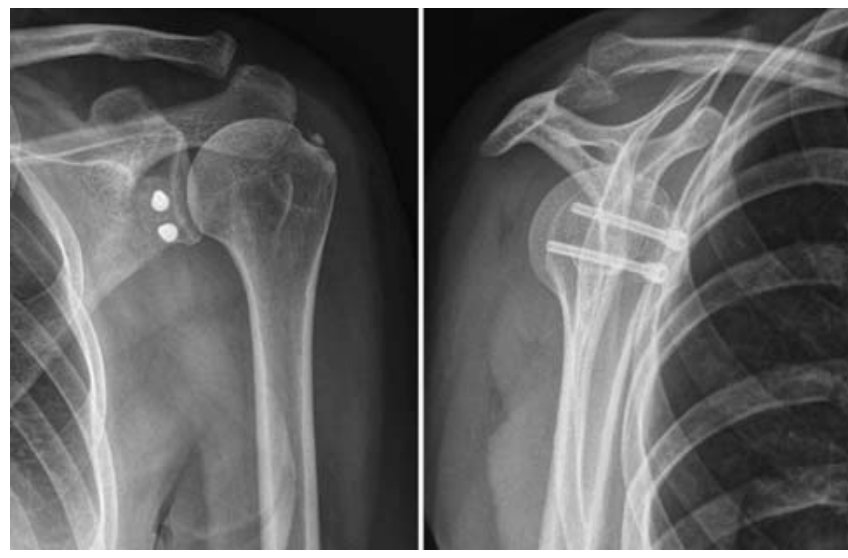

Abb. 17 Röntgenbild nach arthroskopischer Latarjet-Operation bei einer $\mathrm{Pa}$ tientin mit Knochenverlust am vorderen Pfannenrand.

tischen vorderen Schulterinstabilität ist die hintere Instabilität häufig atraumatisch und mit einer multidirektionalen Instabilität vergesellschaftet [6]. Neben Weichteilverletzungen, wie Kapsel-Labrum-Verletzungen, spielen bei dieser Form der Instabilität auch knöcherne Veränderungen am Pfannenrand, muskuläre Fehlsteuerungen oder eine gestörte skapulothorakale Kinematik eine wichtige Rolle.

\section{Indikation}

Patienten mit hinterer Instabilität sprechen besser auf ein intensives Krankengymnastikprogramm an als Patienten mit vorderer Instabilität. Daher sollte zunächst ein konservatives Therapieprogramm mit Kräftigung des M. infraspinatus bei dieser Form der Instabilität gewählt werden. Ein besonderes Augenmerk sollte dabei auch auf die Scapulasteuerung und muskuläre Koordination gerichtet werden. Sollte nach einem Zeitraum von mindestens 6 Monaten keine nachhaltige Besserung der Symptome zu verzeichnen sein, sollten operative Behandlungsmöglichkeiten in Betracht gezogen werden.

\section{Arthroskopische Operationstechnik bei dorsaler Schulterinstabilität}

In Intubationsnarkose werden die Patienten in Seitenlagerung positioniert. Prinzipiell ist auch eine Beach-Chair-Lagerung möglich, allerdings bietet die Seitenlagerung den Vorteil, durch den axialen Längszug und falls nötig zusätzlicher Lateraltraktion ausreichend Gelenkdistraktion und somit bessere Visualisierung aller Gelenkabschnitte zu schaffen. Es sollte zunächst eine ausgiebige Narkoseuntersuchung durchgeführt werden, um das
Ausmaß und die Richtung der Translation des Oberarmkopfs zu überprüfen.

\section{Dorsale Stabilisierung}

Für die dorsale Stabilisierung muss meist ein zusätzliches posteroinferiores Portal angelegt werden, über das ein korrekter Einbring-Winkel für Anker am dorsalen Glenoidrand möglich ist. Dieses Portal liegt $3 \mathrm{~cm}$ distal und $1 \mathrm{~cm}$ medial der posterolateralen Akromionecke. Zunächst wird ein glenohumeraler diagnostischer Rundgang des ventralen Gelenkabschnitts durchgeführt. Neben Kapsel-Labrum-Verletzungen sollte dabei auf das Rotatorenintervall geachtet werden.

Beim Vorliegen einer dorsalen BankartLäsion wird analog zur vorderen Stabilisierung der hintere Labrumkomplex mit dem hinteren Anteil des IGHLs vom Glenoidrand mobilisiert. Die Glenoidkante wird mit einem Bankart-Raspatorium oder einem motorisierten Shaver angefrischt. An die Glenoidkante wird ein Fadenanker in einem $45^{\circ}$-Winkel zur Glenoidlängs- und -querachse über das posterolaterale Portal eingebracht. Im Gegensatz zur Ankerplatzierung am vorderen Glenoid ist die Ankerplatzierung am dorsalen Glenoid aufgrund der knöchernen Geometrie nicht ganz unproblematisch. Der Winkel zwischen der Gelenkfläche und dem hinteren Kortex ist steiler als der Winkel zwischen dem vorderen Kortex und der Gelenkfläche. Anschließend wird das mobilisierte Kapsel-Labrum-Gewebe mit einem kanülierten Nahthaken von geeigneter Krümmung durchstochen und ein Shift nach kranial und medial durchgeführt. Dabei muss das posteriore IGHL perforiert werden, um einen ausreichenden Kapselshift $\mathrm{zu}$ ermöglichen. Anschließend erfolgt 
das stabile Verknoten unter Zuhilfenahme eines arthroskopischen Knotenschiebers. Bei Verwendung des Pushlock-Ankers wird ein gedoppelter Fiberwire-Faden mithilfe des PDS Shuttle Relay durch das mobilisierte Kapsel-Labrum-Gewebe transportiert. Beide Enden des Fadens werden durch die Öse des Ankers außerhalb des Gelenks eingefädelt und diese unter dosiertem Anspannen des Fadenpaars in das zuvor angelegte Bohrloch eingestösselt.

\section{Kapselplikaturen}

Zur Reduktion einer Kapselredundanz wird die hintere Kapsel, die dünner als die vordere ist, zunächst sehr vorsichtig angefrischt, um eine fibroblastische Einheilung zu ermöglichen. Mit kontinuierlichem Flow wird die oberflächliche Synoviaschicht mit dem Synovator oder mit einem Raspartorium angefrischt, indem sie tangential mit gleichmäßigen scheibenwischerartigen Bewegungen ausgestrichen wird. Mit einem kanülierten Nahthaken wird die Kapsel, beginnend in der 6-Uhr-Position, mit einem Abstand von $1 \mathrm{~cm}$ zum Glenoid perforiert, die Nadelspitze ausgestochen, und das nach kranial geraffte Kapselgewebe durch das intakte Labrum durchstochen. Ein monofiler Faden (PDS USP 2-0) wird durch die Nadel geschoben, und der Nahthaken entfernt. Mit einem Fadenholer wird der nun intraartikulär liegende Faden nach posterior ausgeleitet und ein Orthocord-Faden durch das Kapsel-Labrum-Gewebe durchgeshuttelt. Anschließend wird das verkürzte Kapselgewebe unter dosiertem Anspannen unter Zuhilfenahme eines arthroskopischen Knotenschiebers geknüpft. Das Verknoten muss vorsichtig erfolgen, um die dünne Kapsel nicht durchzureißen. Alternativ kann auch ein PDS-USP-0-Faden benützt werden. Mit einem Abstand von $1 \mathrm{~cm}$ werden nun Plikaturen von der 6-Uhr- bis zur 11-Uhr-Position angelegt, bis die hintere Kapselredundanz in allen Bereichen aufgehoben ist. In der Regel sind 4-6 Plikaturen notwendig, um eine suffiziente Kaspelreduktion $\mathrm{zu}$ erreichen. Es empfiehlt sich, nach diesem Schritt erneut eine dynamische Untersuchung durchzuführen, um die veränderte Gelenkkinematik zu kontrollieren. Es sollte unbedingt darauf geachtet werden, nicht zu viel Kapselgewebe zu verkürzen, da ansonsten eine ausgeprägte Innenrotationseinschränkung resultiert.

Bei den multidirektionalen Instabilitätsformen erfolgt nach Portalwechsel in identischer Technik die Anlage von Kapselplikaturen im ventralen Abschnitt. Von anteroinferior beginnend werden Plikaturen im Abstand von $1 \mathrm{~cm}$ zum Glenoid angelegt, bis die ventrale Kapselredundanz aufgehoben ist. Insgesamt sollten ebenfalls 4-6 Plikaturen von der 6-Uhr- bis zur 1-Uhr-Position angelegt werden. Anschließend sollte erneut die korrigierte Gelenkkinematik dynamisch untersucht werden.

Bei Insuffizienz des Rotatorenintervalls, welche sich klinisch in einem vermehrten Sulcuszeichen (Abb. 11) in Neutralstellung und in $20^{\circ}$-Außenrotation zeigt, ist ein Verschluss des Rotatorenintervalls sinnvoll.

Nachbehandlung bei dorsaler

Stabilisierung

Die Patienten sollten zunächst in einer Orthese in $15^{\circ}$ Außenrotation für die ersten beiden postoperativen Wochen immobilisiert werden. Von der 3.-6. Woche kann die Abduktion und Flexion bis $60^{\circ}$ freigegeben werden. Die Außenrotation ist bei den reinen dorsalen Stabilisierungen freigegeben. Die Innenrotation ist bei adduziertem Arm zum Bauch freigegeben. In den ersten 6 Wochen sollten keine forcierten Innenrotationsübungen durchgeführt werden. Nach 6 Wochen kann das Bewegungsausmaß sukzessiv gesteigert werden, bis eine freie glenohumerale Beweglichkeit erreicht wird. Zuletzt sollten auch die Rotatorenmanschette, der M. deltoideus und die Scapulastabilisatoren auftrainiert werden. Überkopf- oder Kontaktsport sollte für die Dauer von 6 Monaten nicht durchgeführt werden.

\section{Knöcherne Defekte}

Die beschriebenen Operationstechniken der weichteiligen Kapsel-Labrum-Rekonstruktion sind bei relevanten knöchernen Defekten am Glenoidrand nicht mehr geeignet.

Durch biomechanische und klinische Studien wurde gezeigt, dass bei einem Verlust des anteroinferioren Glenoidknochens von mehr als $20 \%$ des a.-p. Durchmessers der Knochenverlust durch ein geeignetes Verfahren ausgeglichen werden muss. In unserer Klinik hat sich hierfür der Transfer des Processus coracoideus (sog. Latarjet-Operation) bewährt, der neben dem Aufbau des Glenoidknochens die Schulter durch Umlenkung der am Korakoid inserierenden Sehnen („sling-Effekt“) weichteilig stabilisiert.
Die Indikation für den Korakoidtransfer und Kontraindikation für die BankartOperation ist somit gegeben, wenn ein ausgedehnter knöcherner Defekt an Glenoid (und/oder Humerus), eine nicht reparable Weichteilsituation (z.B. großer chronischer HAGL-Defekt, evidenter IGHL-Defekt, Labrumzerstörung) oder Kombinationen aus diesen Pathologien vorhanden sind.

Darüber hinaus ist die Latarjet-Technik eine sehr gute Option für das Management von Rezidiven nach Bankart-Operationen, insbesondere bei sehr aktiven Patienten und Schultersportlern, bei denen aufgrund der komplexen Pathomorphologie der Instabilität eine erneute Bankart-Operation meist kontraindiziert ist.

Die Operationstechnik kann offen oder bei entsprechender Expertise auch arthroskopisch durchgeführt werden; sie ist von hoher Komplexität, zeigt bei korrekter Durchführung aber exzellente Ergebnisse mit niedrigen Reluxationsraten auch über einen langfristigen Zeitraum.

\section{Literatur}

${ }^{1}$ Agneskirchner JD, Haag M, Lafosse L. Arthroscopic coracoid transfer: indications, technique and initial results. Orthopade 2011; 40: 41-51

2 Balg F, Boileau P. The instability severity index score. A simple pre-operative score to select patients for arthroscopic or open shoulder stabilisation. J Bone Joint Surg [Br] 2007; 89: 1470-1477

${ }^{3}$ Bankart ASB. The pathology and treatment of recurrent dislocation of the shoulder joint. Brit J Surg 1938; 26: 23-29

4 Boileau P. Villalba M, Héry JY et al. Risk factors for recurrence of shoulder instability after arthroscopic Bankart repair. J Bone Joint Surg [Am] 2006; 88: 1755-1763

5 Burkhart SS, De Beer JF. Traumatic glenohumeral bone defects and their relationship to failure of arthroscopic Bankart repairs: significance of the inverted-pear glenoid and the humeral engaging Hill-Sachs lesion. Arthroscopy 2000; 16: 677-694

${ }^{6}$ Burkhart SS et al. Quantifying glenoid bone loss arthroscopically in shoulder instability. Arthroscopy 2002; 18: 488-491

${ }^{7}$ Carreira DS et al. A prospective outcome evaluation of arthroscopic Bankart repairs: minimum 2-year follow-up. Am J Sports Med 2006; 34: 771-777

8 Cheung EV et al. Long-term outcome of anterior stabilization of the shoulder. J Shoulder Elbow Surg 2008; 17: 265-270

9 Elmlund $A O$ et al. A 7-year prospective, randomized, clinical, and radiographic study after arthroscopic Bankart reconstruction using 2 different types of absorbable Tack. Am J Sports Med 2009; 37: 1930-1937

10 Gerber C, Nyffeler RW. Classification of glenohumeral joint instability. Clin Orthop Relat Res 2002; 400: 65-76 
11 Halder AM et al. Effects of the glenoid labrum and glenohumeral abduction on stability of the shoulder joint through concavity-compression: an in vitro study. J Bone Joint Surg [Am] 2001; 83: 1062-1069

12 Helfet AJ. Coracoid transplantation for recurring dislocation of the shoulder. J Bone Joint Surg [Br] 1958; 40: 198-202

13 Hovelius L, Sandstrom B, Saebö M. One hundred eighteen Bristow-Latarjet repairs for recurrent anterior dislocation of the shoulder prospectively followed for fifteen years: study II - the evolution of dislocation arthropathy. J Shoulder Elbow Surg 2006; 15: 279-289

14 Hovelius L et al. One hundred eighteen Bristow-Latarjet repairs for recurrent anterior dislocation of the shoulder prospectively followed for fifteen years: study I - clinical results. J Shoulder Elbow Surg 2004; 13: 509516

15 Huijsmans PE et al. Quantification of a glenoid defect with three-dimensional computed tomography and magnetic resonance imaging: a cadaveric study. J Shoulder Elbow Surg 2007; 16: 803-809

16 Ide J, Maeda S, Takagi K. Arthroscopic Bankart repair using suture anchors in athletes: patient selection and postoperative sports activity. Am J Sports Med 2004; 32: 1899-1905

17 Itoi E et al. Quantitative assessment of classic anteroinferior bony Bankart lesions by radiography and computed tomography. Am J Sports Med 2003; 31: 112-118

18 Itoi $E$ et al. The effect of a glenoid defect on anteroinferior stability of the shoulder after Bankart repair: a cadaveric study. J Bone Joint Surg [Am] 2000; 82: 35-46

$19 \mathrm{Kim} \mathrm{SH}$ et al. Arthroscopic anterior stabilization of the shoulder: two to six-year followup. J Bone Joint Surg [Am] 2003; 85: 15111518

20 Lafosse L, Baier GP, Jost B. Footprint fixation for arthroscopic reconstruction in anterior shoulder instability: the Cassiopeia doublerow technique. Arthroscopy 2006; 22: 231 e1-231.e6
21 Lafosse L, Lejeune E, Bouchard A et al. The arthroscopic Latarjet procedure for the treatment of anterior shoulder instability. Arthroscopy 2007; 23: 1242.e1-1242.e5

22 Latarjet $M$. Treatment of recurrent dislocation of the shoulder. Lyon Chir 1954; 49: 994-997

23 Lazarus MD et al. Effect of a chondral-labral defect on glenoid concavity and glenohumeral stability. A cadaveric model. J Bone Joint Surg [Am] 1996; 78: 94-102

24 Magnusson L, Kartus J, Ejerhed L et al. Revisiting the open Bankart experience: a four- to nine-year follow-up. Am J Sports Med 2002; 30: 778-782

${ }^{25}$ Mohtadi NG, Bitar IJ, Sasyniuk TM et al. Arthroscopic versus open repair for traumatic anterior shoulder instability: a meta-analysis. Arthroscopy 2005; 21: 652-658

${ }^{26}$ Neviaser TJ. The anterior labroligamentous periosteal sleeve avulsion lesion: a cause of anterior instability of the shoulder. Arthroscopy 1993; 9: 17-21

27 O'Connell PW, Nuber GW, Mileski RA et al. The contribution of the glenohumeral ligaments to anterior stability of the shoulder joint. Am J Sports Med 1990; 18: 579-584

28 Owens BD, DeBerardino TM, Nelson BJ et al. Long-term follow-up of acute arthroscopic Bankart repair for initial anterior shoulder dislocations in young athletes. Am J Sports Med 2009; 37: 669-673

29 Patte D, Rodineau J, Gardes JC. Epaules douloureuses et instables. Rev Chir Orthop 1980; 66: 157-165

30 Provencher MT, Detterline AJ, Ghodadra N et al. Measurement of glenoid bone loss: a comparison of measurement error between 45 degrees and 0 degrees bone loss models and with different posterior arthroscopy portal locations. Am J Sports Med 2008; 36: 11321138

31 Purchase RJ et al. Hill-Sachs "remplissage": an arthroscopic solution for the engaging HillSachs lesion. Arthroscopy 2008; 24: 723-726

32 Rowe CR, Patel D, Southmayd WW. The Bankart procedure: a long-term end-result study. J Bone Joint Surg [Am] 1978; 60: 1-16
33 Sugaya H, Moriishi J, Dohi M et al. Glenoid rim morphology in recurrent anterior glenohumeral instability. J Bone Joint Surg [Am] 2003; 85: 878-884

${ }^{34}$ Tjoumakaris FP, Abboud JA, Hasan SA et al. Arthroscopic and open Bankart repairs provide similar outcomes. Clin Orthop Relat Res 2006; 446: 227-232

35 Wellmann M, Petersen W, Zantop T et al. Open shoulder repair of osseous glenoid defects: biomechanical effectiveness of the Latarjet procedure versus a contoured structural bone graft. Am J Sports Med 2009; 37: 87-94

\section{Dr. med. Sanjay Weber-Spickschen} Assistenzarzt

Sportsclinic Germany Uhlemeyerstraße 16 30175 Hannover

\section{Dr. med. Hamid Hosseini}

Geschäftsführender Oberarzt

Klinik für Unfall- und Wiederherstellungschirurgie Diakoniekrankenhaus Henriettenstiftung Hannover

Priv.-Doz. Dr. med. Jens Agneskirchner Leitender Arzt

Sportsclinic Germany Uhlemeyerstraße 16 30175 Hannover

jens.agneskirchner@ sportsclinicgermany.com 